\title{
Maize Plants Chimeric for an Autoactive Resistance Gene Display a Cell-Autonomous Hypersensitive Response but Non-Cell Autonomous Defense Signaling
}

\author{
Shailesh Karre, ${ }^{1 \dagger}$ Saet-Byul Kim, ${ }^{1}$ Bong-Suk Kim, ${ }^{2}$ Rajdeep S. Khangura, ${ }^{3}$ Shannon M. Sermons, ${ }^{1,4}$ \\ Brian Dilkes, ${ }^{3}$ Guri Johal, ${ }^{2}$ and Peter Balint-Kurti ${ }^{1,4, \dagger}$ \\ ${ }^{1}$ Department of Entomology and Plant Pathology, North Carolina State University, Raleigh, NC 27695-7613, U.S.A. \\ ${ }^{2}$ Botany and Plant Pathology, Purdue University, West Lafayette, IN, U.S.A. \\ ${ }^{3}$ Department of Biochemistry, Purdue University, West Lafayette, IN, U.S.A. \\ ${ }^{4}$ Plant Science Research Unit, United States Department of Agriculture-Agricultural Research Service, North Carolina State \\ University, Raleigh, NC 27695-7613, U.S.A.
}

Accepted 27 January 2021.

\begin{abstract}
The maize gene $R p 1-D 21$ is a mutant form of the gene $R p 1-D$ that confers resistance to common rust. $R p 1-D 21$ triggers a spontaneous defense response that occurs in the absence of the pathogen and includes a programed cell death called the hypersensitive response (HR). Eleven plants heterozygous for $R p 1-D 21$, in four different genetic backgrounds, were identified that had chimeric leaves with lesioned sectors showing HR abutting green nonlesioned sectors lacking HR. The Rp1-D21 sequence derived from each of the lesioned portions of leaves was unaltered from the expected sequence whereas the Rp1-D21 sequences from nine of the nonlesioned sectors displayed various mutations, and we were unable to amplify Rp1-D21 from the other two nonlesioned sectors. In every case, the borders between the sectors were sharp, with no transition zone, suggesting that HR and chlorosis associated with $R p 1-D 21$ activity was cell autonomous. Expression of defense response marker genes was assessed in the lesioned and nonlesioned sectors as well as in near-isogenic plants lacking and carrying $R p 1-D 21$. Defense gene expression was somewhat elevated in nonlesioned sectors abutting sectors carrying $R p 1-D 21$ compared with near-isogenic plants lacking $R p 1-D 21$. This suggests that, whereas the HR itself was cell autonomous, other aspects of the defense response initiated by $R p 1-D 21$ were not.
\end{abstract}

Keywords: cell death, plant responses to pathogens, resistance genes

${ }^{\dagger}$ Corresponding authors: S. Karre; skarres@ncsu.edu and P. Balint-Kurti; Peter.Balint-Kurti@USDA.GOV

Funding: This work was supported by the United States Department of Agriculture-Agricultural Research Service, North Carolina State University, and National Science Foundation Plant Genome grants 0822495 and 1444503 .

*The $\boldsymbol{e}$-Xtra logo stands for "electronic extra" and indicates that supplementary materials are published online.

The author(s) declare no conflict of interest.

(c) (1) () $\odot$ Copyright $(2021$ The Author(s). This is an open access article (c) 1 (8)
The hypersensitive response (HR) is a rapid localized cell death in response to pathogen detection (Balint-Kurti 2019; Mur et al. 2008). It provides effective resistance to a wide range of biotrophic pathogens, including fungi, oomycetes, bacteria, and viruses, as well as other organisms such as insects (Rossi et al. 1998) and nematodes (Dropkin 1969) that form sustained intimate interactions with the host plant. It is generally less effective against, and may actually be beneficial to, necrotrophs, which require dead host tissue to complete their life cycle (Virdi et al. 2016).

The term HR has been used to describe a number of situations involving localized necrosis (Balint-Kurti 2019). Typically, HR is closely associated with gene-for-gene interactions (Flor 1942), in which a dominant resistance $(R)$ gene in the host corresponds with a dominant avirulence (Avr) gene in the pathogen. Resistance, usually involving $\mathrm{HR}$, is only conferred if both the $R$ gene and the corresponding $A v r$ gene are present in the same interaction. $A v r$ genes are now understood to encode effectors: molecules secreted by the pathogen into the host to enable the pathogenesis process (Hogenhout et al. 2009). Avr proteins are simply effectors whose presence can be detected by specific R-proteins leading to the activation of the defense response.

Most $R$ genes encode cytoplasmic-located proteins possessing both a nucleotide-binding site (NBS) or nucleotide-binding adaptor shared by APAF-1, R proteins, and CED-4 (NB-ARC) and leucine-rich (LRR) repeat domains known as NBS-LRR proteins (NLRs). Most NLRs carry either coiled-coil (CC) or toll-interleukin receptor (TIR) domains at their $\mathrm{N}$ terminus. Although similarities in NLR structure and function suggest a high level of conservation of mechanism, this is not necessarily the case. Notably, the mechanisms of their activation vary considerably (Kourelis and van der Hoorn 2018; Wang and Balint-Kurti 2015). In general, the $\mathrm{N}$-terminal domains are responsible for activating cell death pathways while the NBS and LRR domains regulate the activity of the NLR and auto-inhibit activation through intraprotein interactions (Kourelis and van der Hoorn 2018; Wang et al. 2015b). NLR activity is additionally inhibited via a range of interactions with other proteins (Balint-Kurti 2019).

In a recent groundbreaking study, Wang et al. (2019a and b) demonstrated that, upon activation via indirect interaction with its cognate Avr protein, the Arabidopsis thaliana CC-NLR ZAR1 forms a homopentamer which relocates to the plasma membrane. Structural modeling suggested that the $\mathrm{CC}$ domains of the five subunits form a funnel-shaped structure which may be capable of creating pores in the plasma membrane to initiate the defense 
response and HR. In other work, two TIR-NLRs, RPP1 and ROQ1 from Arabidopsis and Nicotiana benthamiana, respectively, were shown to form tetrameric structures upon direct binding of their cognate effectors (Ma et al. 2020; Martin et al. 2020; Tian and Li 2020). These findings, that activation of NLRs involve oligomerization into a so-called "resistosome", are likely to be broadly applicable (Dangl and Jones 2019) but it is still not clear how these NLRs activate the defense response or if they all do so in a similar manner. Signal transduction is thought to involve the N-terminal domains. Structural modeling suggested that the CC domains of the five subunits form a funnel-shaped structure which may be capable of creating pores in the plasma membrane to initiate the defense response and HR (Wang et al. 2019a and b). TIR domains of NLRs were shown to possess an $\mathrm{NAD}^{+}$-cleaving activity required for cell death (Horsefield et al. 2019; Wan et al. 2019). In some cases, NLRs translocate to the nucleus upon activation and interact with transcription factors (Chang et al. 2013).

The rpl locus is a complex locus in the maize genome carrying multiple tandemly repeated CC-NLR paralogs that confer resistance to specific races of the fungus Puccinia sorghi, the causal agent of common rust (Hulbert 1997). Diverse rpl haplotypes have been identified and at least 16 distinct race-specific resistances have been mapped to this locus (Hulbert 1997). The rpl locus is notoriously unstable, and unequal crossing over between CCNLR genes within rpl (Hulbert and Bennetzen 1991; Sudupak et al. 1993) sometimes results in intragenic recombination (i.e., the recombination of the $5^{\prime}$ end of one paralog with the $3^{\prime}$ end of another, to produce a chimeric gene). Rp1-D21 is one such allele that resulted from intragenic recombination between the Rp1 paralogs Rpl-D and Rpl-dp2. The protein encoded by Rpl-D21 is spontaneously activated without the need for pathogen recognition and causes HR lesions on leaves of maize plants in the absence of pathogen ( $\mathrm{Hu}$ et al. 1996; Wang et al. 2015b). The severity of the HR phenotype conferred by Rpl-D21, and the associated stunting of growth, is genetic background dependent (Chintamanani et al. 2010; Negeri et al. 2013).

The mechanism of activation of Rp1-D21, the loci affecting the severity of the HR phenotype it confers, and the proteins and pathways it interacts with have been the subject of a number of studies (Chaikam et al. 2011; Chintamanani et al. 2010; He et al. 2019a and b; Murphree et al. 2020; Negeri et al. 2013; Olukolu et al. 2013, 2014; Wang and Balint-Kurti 2016, 2015; Wang et al. 2015a and b), and several of the mechanisms by which Rp1-D21 is regulated have been partially elucidated. However, using this system, it has not been possible to unambiguously investigate the relationships between cells in which Rpl-D21 is activated and those in which it is not.

Chimeric, or genetic mosaic, plants have been used extensively in the study of plant development (Freeling 1992; Scanlon and Freeling 1997). These plants carry juxtaposed sectors that differ for one or more genes, and this allows the investigation of the cell-autonomous nature of the action of these genes and their role in signaling between cells (Fyvie et al. 2000). The only reported use of genetic mosaics to investigate R-protein action involved Rpl. Bennetzen et al. (1988) used X-ray treatment of seed heterozygous for Rpl to generate leaf sectors with chromosome breaks on the short arm of chromosome 10. These sectors lacked the oil yellow $(O y)$ gene which caused the sectors to be yellow. The yellow sectors were also predicted to lack $R p l$ because $R p l$ is distal to $O y$ on chromosome 10. P. sorghi was able to infect the yellow sectors and produce pustules, whereas infection of green parts of the plant resulted in HR. This occurred even in cases where the yellow sectors were only six cells wide. HR initiated in green sectors that bordered yellow sectors appeared to propagate a short distance into the yellow sectors but propagated much more extensively in the green sectors. Overall, the authors stated that these results demonstrated that "the initiation of the hypersensitive response and resistance to $P$. sorghi require a cell-autonomous non-diffusible factor specified by $R p l$ ". They also reported that the $R p l$ gene was likely involved in "propagation as well as initiation of the necrotic response".

Jones and Deverall (1978) used a temperature sensitive R protein and a "leaf transplant" technique to mechanically create wheat genetic mosaics to investigate the cell autonomy of HR. The epidermis of infected and uninfected wheat leaves was peeled away, and the mesophyll cell layers of both leaves were then brought into contact and held together for several days. By using cultivars with and without the $L r 20$ P. recondita $R$ gene, they were able to show that necrosis (presumably caused by HR) occurred in uninfected tissue carrying $\operatorname{Lr} 20$ when it was brought into contact with tissue infected with $P$. recondita that was avirulent on cultivars carrying $\operatorname{Lr} 20$. The genotype of the infected tissue did not affect the presence or absence of necrosis in the uninfected tissue. This suggested that $L r 20$ gene action was cell autonomous because the presence of $\operatorname{Lr} 20$ in the infected tissue was not enough to induce HR in the uninfected tissue. It also suggested that $\operatorname{Lr} 20$ was required for the propagation of the HR. Although the molecular identity of the Lr2O gene is not yet described, it is likely to be an NLR because it is a dominant gene (Browder 1972) conferring disease resistance associated with an HR.

Therefore, the findings of both the Jones and Deverall (1978) and the Bennetzen et al. (1988) studies are largely similar in suggesting that $R$ gene action is largely cell autonomous and that $R$ genes are required for the activation and the propagation of a HR. In this study, we were able to use updated techniques to confirm and extend these findings. We used spontaneously occurring and ethyl methanesulfonate (EMS)-generated chimeric plants to show that the HR induced by Rp1-D21 is cell autonomous, though some aspects of the defense response signaling are not. We identified a set of mutations in the Rp1-D21 gene capable of suppressing or abrogating activity and provide evidence that $R p 1-D 21$ is activated in most cells in which is it present, not only the cells displaying HR cell death.

\section{RESULTS AND DISCUSSION}

Identification of plants with chimeric leaves suggests that the HR phenotype conferred by $R p 1-D 21$ is cell autonomous.

Approximately 50,000 plants carrying Rpl-D21 were observed in the summer of 2017, as part of a related study. Among these, two chimeric plants carrying leaf sectors of nonlesioned tissue abutting tissue displaying HR-induced lesions were identified. Chimeric mutant 1 (CM1) was derived from a Tx303 $\times$ H95:Rp1-D21 cross and chimeric mutant 2 (CM2) was derived from a M37W $\times$ H95:Rp1-D21 cross (Fig. 1A). These chimeras apparently resulted from spontaneous inactivation of $R p 1-D 21$.

Two independent attempts were made to develop more chimeric mutant plants displaying HR-induced lesions in different maize genetic background. The $R p 1-D 21 \# 4$ allele is a previously described weak allele of Rp1-D21 derived from an EMS mutagenesis screen (Wang et al. 2015b) of previously unknown molecular nature. The B73:Rp1-D21\#4 line is the maize line B73 carrying an introgression of Rp1-D21\#4 maintained in a heterozygous state. EMS mutagenesis of 500 B73:Rp1-D21\#4 seed yielded 11 chimeric plants displaying sectors of nonlesioned tissue on their leaves. Three of these plants had sectors large enough to isolate tissue sufficient for molecular analysis. These plants were referred as chimeric mutants 3-1, 3-2, and 3-3 (CM3-1, CM3-2, and CM3-3) (Fig. 1A). The H95:Rp1-D21 line is the maize line $\mathrm{H} 95$ carrying an introgression of Rpl-D21 maintained in a heterozygous state. Among 1,100 EMS mutagenized H95:Rp1-D21 seeds, 18 chimeric plants displaying nonlesioned sectors were identified. Six of these plants showing the 
clearest sectors were selected for further study. These plants were named chimeric mutants 4-1, 4-5, 4-6, 4-10, 4-12, and 4-13 (CM4-1, CM4-5, CM4-6, CM4-10, CM4-12, and CM4-13).

In every case, regardless of size or orientation, the border between the lesioned and nonlesioned tissue was abrupt, with no transition zone, and this was apparent at both the macroscopic and microscopic levels (Fig. 1A and B), suggestive of a cell-autonomous phenotype. All patterns were consistent with mitotic transmission of the sector. Also notable was the fact that many living (nonbrown) cells in the lesioned sector were significantly lighter green or chlorotic compared with the nonlesioned sector. This chlorosis was also entirely confined to the lesioned sector with a clear demarcation at the sector boundary, indicating that the chlorosis phenotype was cell autonomous also (Fig. 1A, magnified insets). The fact the cells close to the border in the nonlesioned sectors did not display any chlorosis strongly suggests that the chlorosis observed in the lesioned sector is due to the effects of Rp1-D21 in those cells rather than the fact that they are adjacent to dead cells. This suggests that Rpl-D21 is activated in all or most of the cells in the lesioned sectors. Within the lesioned areas, cells varied from light green to yellow to brown and necrotic. The patches of lightgreen cells in the lesioned sectors may indicate areas in Rp1-D21 has not yet been activated or has only recently activated, or where negative regulation of HR has maintained viability.

Analysis of cell death marker gene expression.

Cell death marker genes such as the hairpin-induced gene (ZmHinl) and EF-hand Ca2+-binding protein (ZmCCD1)
(Pontier et al. 1999; Takezawa 2000) have been shown to be induced during HR cell death in several studies (Christensen et al. 2015; Yeom et al. 2012). Quantitative real-time PCR (qRTPCR) analysis of CM1, CM2, and CM3 plants showed that the transcript levels of ZmHinland ZmCCDl were significantly higher in lesioned sectors and nonchimeric isogenic lesioned plants carrying Rpl-D21 than in nonlesioned sectors and wildtype isogenic plants lacking the $R p 1-D 21$ gene, here referred to as nonlesioned plants (Fig. 2). No increased expression was observed in nonlesioned sectors compared with nonlesioned plants. In the case of ZmHinl, we were unable to amplify the gene from either lesioned or nonlesioned sectors of CM3 plants. We suspect that this may be associated with the age of the plants when sampled (70 days after planting for CM1 and CM2 and 28 days for CM3). These results support the conclusion that the HR induced by $R p 1-D 21$ is a cell-autonomous phenotype.

Spontaneous mutations in $R p 1-D 21$ gene led to formation of nonlesioned chimeric sectors and confirm cell-autonomous action of $R p 1-D 21$.

We attempted to amplify and sequence the Rpl-D21 gene from lesioned and nonlesioned sectors of the 11 chimeric mutants using both Wideseq and Sanger sequencing approaches (Table 1). As expected, in all of the lesioned sectors from CM1, CM2, and CM4, the Rp1-D21 (Wang et al. 2015b) sequence was recovered. The lesioned sectors of CM3-1, CM3-2, and CM3-3 carried the Rp1-D21\#4 allele, which we here identify for the first time as an $\mathrm{A}_{3614} \mathrm{~T}$ single-nucleotide polymorphism
A

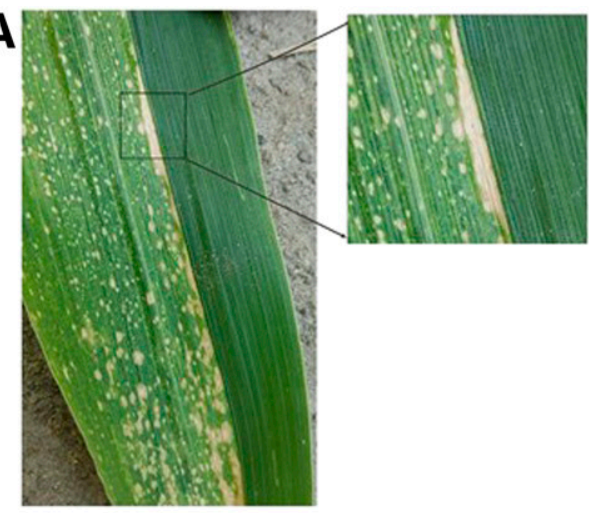

CM1

(T×303 x H95:Rp1D21 M1E1)
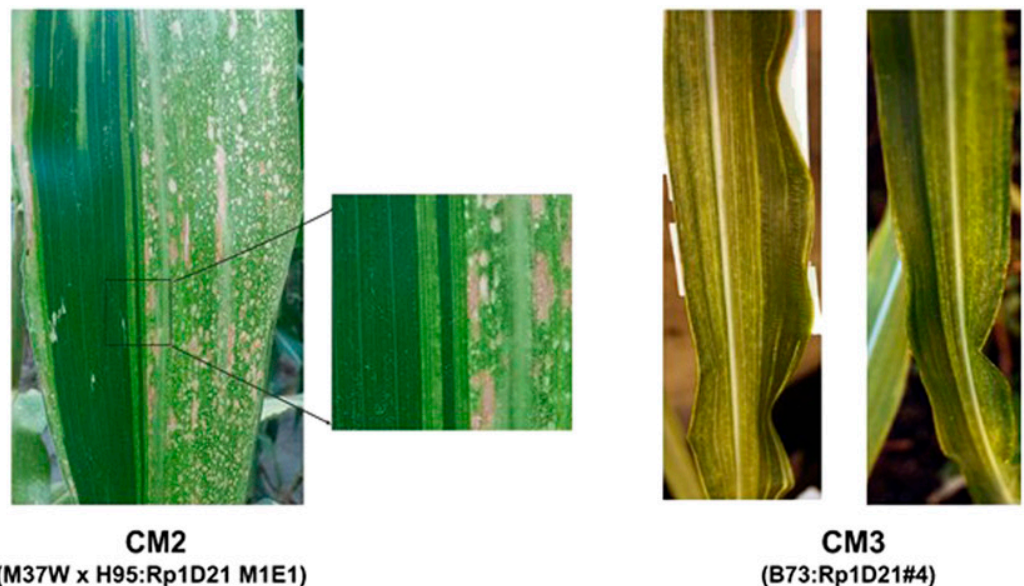

CM3

(B73:Rp1D21\#4)

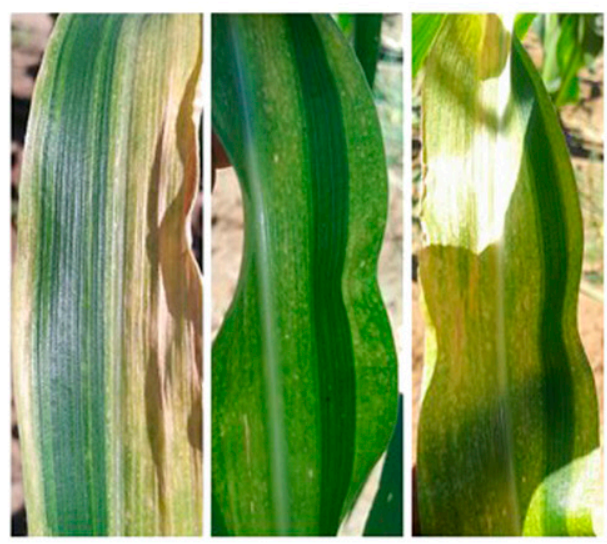

B

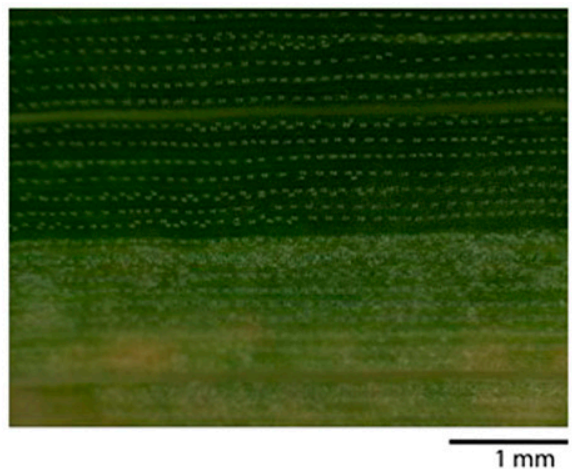

CM4

(H95:Rp1D21)

Fig. 1. Clear demarcation between lesioned and nonlesioned sectors in the chimeric Rp1-D21 plants. A, Macroscopic images of chimeric mutant (CM) sectors showing clear demarcation between lesioned and nonlesioned sectors. Representative CM3 and CM4 leaves are shown. B, Microscopic image of boundary between lesioned and nonlesioned sectors of a representative chimeric mutant showing clear demarcation between sectors. 
(SNP) relative to Rp1-D21 corresponding to an M1205K polymorphism at the protein level (Fig. 3; Supplementary Fig. S2). This is the only missense mutation and the likely cause of the reduced severity of the $R p 1-D 21 \# 4$ mutant.

The Rp1-D21 sequence derived from the nonlesioned sectors of CM1 contained novel alleles of encoded an $\mathrm{A}_{3152} \mathrm{~T}$ SNP relative to $R p 1-D 21$, which is predicted to cause a $\mathrm{V}_{1051} \mathrm{D}$ missense mutation. The CM3-1 nonlesioned sector had a G to A transition relative to $R p 1-D 21 \# 4$ at position 244 , resulting in a missense mutation encoding the change $\mathrm{D} 82 \mathrm{~N}$ in the resulting protein (Fig. 3; Supplementary Fig. S3) that alters a residue in the conserved EDVID motif in the CC domain of Rp1-D21\#4. This same change was previously recovered as a germinal suppressor of HR caused by Rpl-D21 (Wang et al. 2015b). The CM3-3 nonlesioned sector had a $\mathrm{G}$ to $\mathrm{A}$ transition in Rpl-D21\#4 at position 3,013, resulting in an A1005T change within the LRR domain of Rp1-D21 (Fig. 3; Supplementary Fig. S3).

Wideseq results suggested that the Rpl-D21 allele in the CM2 nonlesioned sectors had a $\mathrm{C}_{680} \mathrm{~A}$ substitution in the Rp1-D21 sequence predicted to cause $\mathrm{T}_{227} \mathrm{~N}$ missense mutation, and a large deletion in the LRR domain. However, we were unable to confirm this mutation using a Sanger sequencing approach despite numerous attempts. Similarly, Wideseq data suggested that the CM3-2 nonlesioned sector carried a $\mathrm{C}$ to $\mathrm{T}$

Table 1. Mutations identified in nonlesioned sectors of chimeric plants ${ }^{\mathrm{a}}$

\begin{tabular}{llc}
\hline Plant & \multicolumn{1}{c}{$\begin{array}{c}\text { Single-nucleotide } \\
\text { polymorphism }\end{array}$} & $\begin{array}{c}\text { Wideseq sequences } \\
\text { with mutation (\%) }\end{array}$ \\
\hline CM1 & $\mathrm{A}_{3152} \mathrm{~T}$ & 99.8 \\
CM3-1 & $\mathrm{G}_{244} \mathrm{~A}$ & 51.0 \\
CM3-3 & $\mathrm{G}_{3013} \mathrm{~A}$ & 94.5 \\
CM4-1 & $\mathrm{T}_{3574} \mathrm{C}$ & - \\
CM4-5 & Deletion of T at 3557 & - \\
CM4-6 & $\mathrm{C}_{1903} \mathrm{~T}, \mathrm{~A}_{2507} \mathrm{G}$ & - \\
CM4-10 & $\mathrm{A}_{587} \mathrm{G}$ & - \\
CM4-12 & $\mathrm{G}_{2263} \mathrm{~A}$ & - \\
$\mathrm{CM} 4-13$ & $\mathrm{~A}_{2155} \mathrm{G}, \mathrm{A}_{2453} \mathrm{G}$ & - \\
\hline
\end{tabular}

${ }^{a}$ Mutations in chimeric mutant 2 (CM2) and CM3-2 were not verified by Sanger sequencing and, therefore, are not shown in the table. Mutations in CM1, CM3-1, and CM3-3 were identified by Wideseq and Sanger sequencing. For these mutations, the percentages of sequences from nonlesioned sectors carrying mutations are shown.

\section{ZmCCD1}

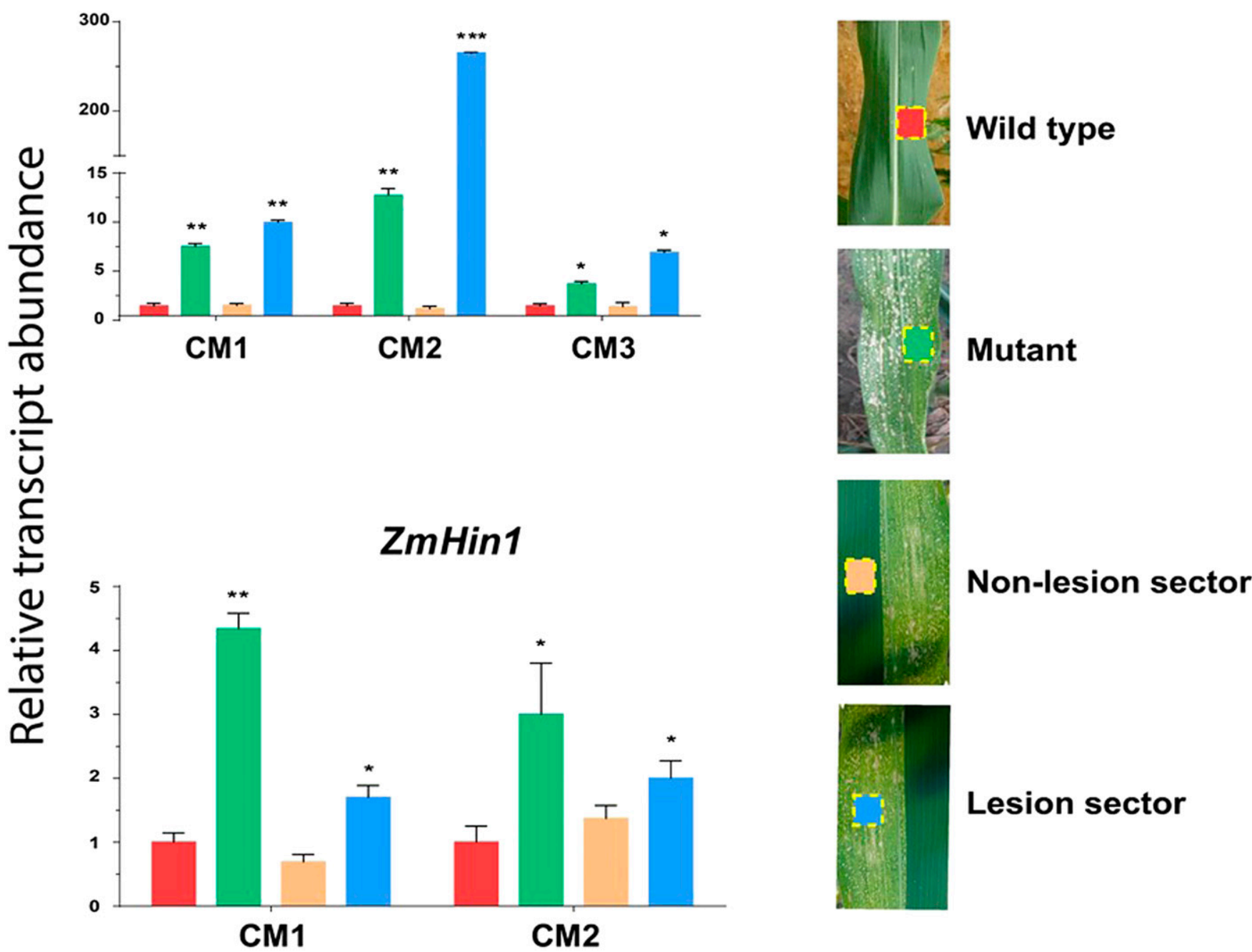

Fig. 2. Expression analysis of cell death marker genes. Quantitative reverse-transcription (qRT)-PCR of cell death marker genes ZmCCD1 and ZmHin1 in nonsectored leaves of plants carrying Rp1-D21 (mutant) and in lesioned sectors of chimeric mutant 1 (CM1), CM2, and CM3. Histogram bars are color coded to indicate type of tissue. A visual key is shown on the right. Results displayed are the averages of all biological replicates in each case. For CM1 and CM2, three biological replicates we collected as individual leaves from the same plant as described. For CM3 qRT-PCR results, we considered samples from CM3-1, CM3-2, and CM3-3 to be three biological replicates. The error bar shows standard error. Significance between treatments were analyzed using Student's $t$ test (asterisks $\left[{ }^{*},{ }^{*},{ }^{* * *}\right]$ indicate $P<0.05,0.01$, and 0.001 , respectively). 
transition relative to $R p 1-D 21 \# 4$ at position 2,308 encoding a change of P770S in the LRR domain. However, as with the $\mathrm{CM} 2$ nonlesioned sector, we were unable to verify this mutation using Sanger sequencing. The Wideseq approach relies on the assembly of multiple short sequences generated using MiSeq. Because the rpl locus is complex, carrying multiple similar homologous sequences, an approach of this type may result in the misassembly of sequences from different homologs. For this reason, in Table 1 and Fig. 3, we are only reporting sequences verified using a Sanger sequencing approach. Nevertheless, we are confident that the Rpl-D21 gene is mutated and nonfunctional in the CM2 and CM3-2 nonlesioned sector due both to the clear visible phenotype and the fact that we also did not detect "wild-type" Rp1-D21 or Rp1-D21\#4 sequences as we did in all of the lesioned sectors of all 11 chimeric plants investigated.

The Rp1-D21 sequence from the CM4-1 nonlesioned sector had a $\mathrm{T}$ to $\mathrm{C}$ transition at position 3,574 , resulting in a P1192S amino acid change in the LRR domain of Rp1-D21. The nonlesioned CM4-5 sector had a deletion of $\mathrm{T}$ at position 3,556 leading to a frame-shift mutation, resulting in a truncated protein. The CM4-6 nonlesioned sector carried two missense mutations in the

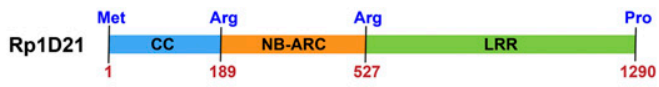

Rp1D21\#4
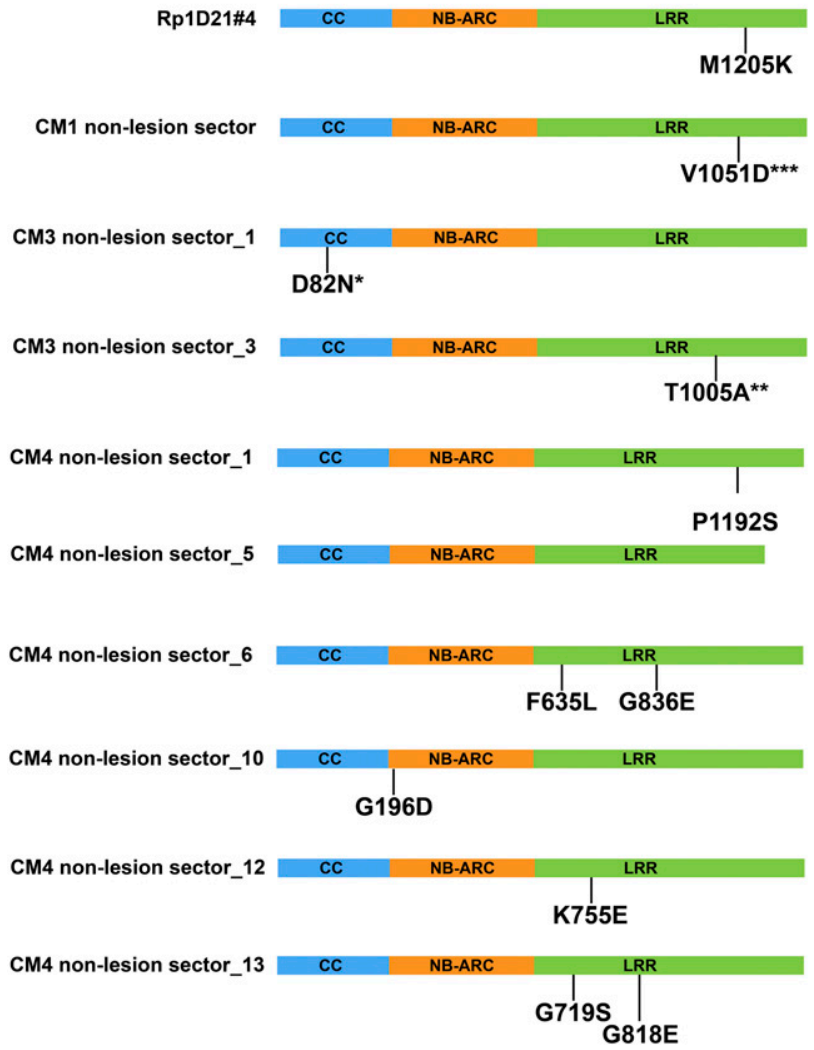

Fig. 3. Mutations causing loss of hypersensitive response (HR) phenotype in nonlesioned sectors. Three domains of the Rp1-D21 protein-the coiled-coil domain; nucleotide-binding adaptor shared by APAF-1, R proteins, and CED-4 domain; and leucine-rich repeat-are indicated by blue, orange, and green shading, respectively. Predicted amino acid polymorphisms identified are denoted below each sequence. Rp1-D21 sequences from chimeric mutant 1 (CM1), CM4-1, CM4-5, CM4-6, CM4-10, CM4-12, and CM4-13 nonlesioned sectors are compared with the reported $R p 1-D 21$ sequence while Rp1D21\#4 is used as reference to compare sequences derived from CM3-1 and CM3-3 nonlesioned sectors. Only sequences verified by Sanger sequencing are shown. One asterisk $\left(^{*}\right)=$ previously shown by Wang et al. $\left(2015\right.$ b) and two $\left(^{* *}\right)=$ transient expression in $N$. benthamiana confirmed no HR induced.
LRR domain of Rpl-D21 gene. The first was a $\mathrm{C}$ to $\mathrm{T}$ transition at position 1,903, resulting in an F635L at the protein level. The second was an $\mathrm{A}$ to $\mathrm{G}$ transition at position 2,507, causing a G836E amino acid change. The Rpl-D21 sequence derived from nonlesioned sector of CM4-10 had an A to G transition at position 587 encoding a G196D change in the NB-ARC domain close to the border between CC and NB-ARC domains. The CM4-12 nonlesioned sector had a $G$ to A transition at position 2,263 which led to change in K755E in the LRR domain. The CM4-13 nonlesioned sector carried two missense mutations in the LRR domain. The first was at position 2,155, where an A to $G$ transition resulted in change of G719S at the protein level, and the second was at position 2,453, where an A to $G$ transition led to a G818E change (Fig. 3). Detailed sequence alignments are shown in Supplementary Figures S1 to S4.

The facts that all of the lesioned sectors carried "normal" Rp1-D21 or Rp1-D21\#4 sequences; that, in nine of the nonlesioned sectors, we identified mutated versions of $R p 1-D 21$ or Rp1-D21\#4; and that, in the other two nonlesioned sectors, we did not identify Rp1-D21 or Rp1-D21\#4 sequences; together with the observation that the sector boundaries are abrupt (Fig. 1), confirm that the HR induced by Rpl-D21 is cell autonomous.

The CM1 and CM2 sectors were caused by spontaneous mutations while the nine CM3 and CM4 sectors were identified on plants treated with the EMS mutagen. The large majority of mutations caused by EMS are G/C to A/T (Kim et al. 2006). Therefore, it is notable that 6 of the 10 mutations we observed in the CM3 and CM4 nonlesioned sectors were not the expected $\mathrm{G} / \mathrm{C}$ to $\mathrm{A} / \mathrm{T}$ transitions. It has been noted previously that the $R p 1$ locus is highly unstable due to its complex nature, with frequent mutations associated with meiotic unequal crossover events (Sudupak et al. 1993); thus, some of the observed Rp1-D21 loss-of-function mutations may not have been related to EMS. EMS can also induce double strand breaks (Ma et al. 2011; Sega 1984; Wagner et al. 2003), which may be repaired by a combination of base excision repair and homologous repair (Klapacz et al. 2016), and it can also induce gross chromosomal abnormalities (Sato and Gaul 1967). It is likely that in the context of the complex tandemly repeated rpl locus, double strand breaks caused by EMS may be repaired using paralogous sequences as templates, inducing more complex mutations as well as simple point mutations in somatic cells.

Previously, Wang et al. (2015b) had identified 12 missense and two nonsense mutations in Rp1-D21 that abrogated the spontaneous HR phenotype. The 12 missense mutants were distributed among the domains: five in the CC, three in the NB-ARC, and four in the LRR domain. Of the 11 mutations identified here, nine were in the LRR, one in the CC domain, and one in the NB-ARC domain. Although the LRR is the largest domain and, therefore, the most likely to be mutated by chance, this distribution is a little surprising. The CC domain is responsible for causing HR while the NB and LRR domains play roles in activation and intraprotein interactions that inhibit activity (Wang et al. 2015b); thus, it would have seemed likely more of mutations suppressing HR would occur in the CC domain.

The maize leaf develops as five tissue layers: two outer epidermal cell layers, a central layer, and two layers in between these (Freeling 1992). As the leaf develops, the middle three layers intermingle so that three developmental layers can be distinguished (Freeling 1992). Depending on the precise position of the cell carrying the original mutation in the meristem, chimeric leaf sectors may carry a mutation only in certain tissue layers or in all of them (Becraft et al. 1990; Freeling 1992; Scanlon and Freeling 1997). The Wideseq technique used to sequence Rp1-D21 from the CM1, CM2, and CM3 nonlesioned sectors provided high sequence coverage (189 to $4,847 \times$ ) (Supplementary Table S2). In order to determine the structures of the chimeras in this study, we 
analyzed the sequencing data derived from each sector to determine the proportion of sequences carrying the mutated allele. If a significant proportion of the sequences from the nonlesioned sectors carried the original allele, that would indicate that the sector was composed of genetically distinct layers. Of the three mutations identified by Wideseq and confirmed by Sanger sequencing shown in Table 1, two were found in more than $90 \%$ of the sequences (Table 1), indicating that they are likely present in all developmental tissue layers. By contrast, the $\mathrm{G}_{244} \mathrm{~A}$ from CM3-1 was identified in $51 \%$ of the sequences, suggesting that it is likely they are not present in all tissue layers.

\section{Ectopic expression of the CM3-3 Rp1-D21 allele} does not induce cell death.

To validate the effect of one of the mutations identified in the Rp1-D21 gene, a transient expression study in $N$. benthamiana was performed. We have previously shown that the HR phenotype conferred by Rpl-D21 in maize can be effectively recapitulated in this system (Wang et al. 2015b). Phenotypic observations at $72 \mathrm{~h}$ postinoculation (hpi) (Fig. 4A) shows that the Rp1-D21 protein encoded by the CM3-3 allele failed to induce HR whereas the functional Rp1-D21 protein derived induced HR as expected. Furthermore, ion leakage analysis confirms this result (Fig. 4B).
A

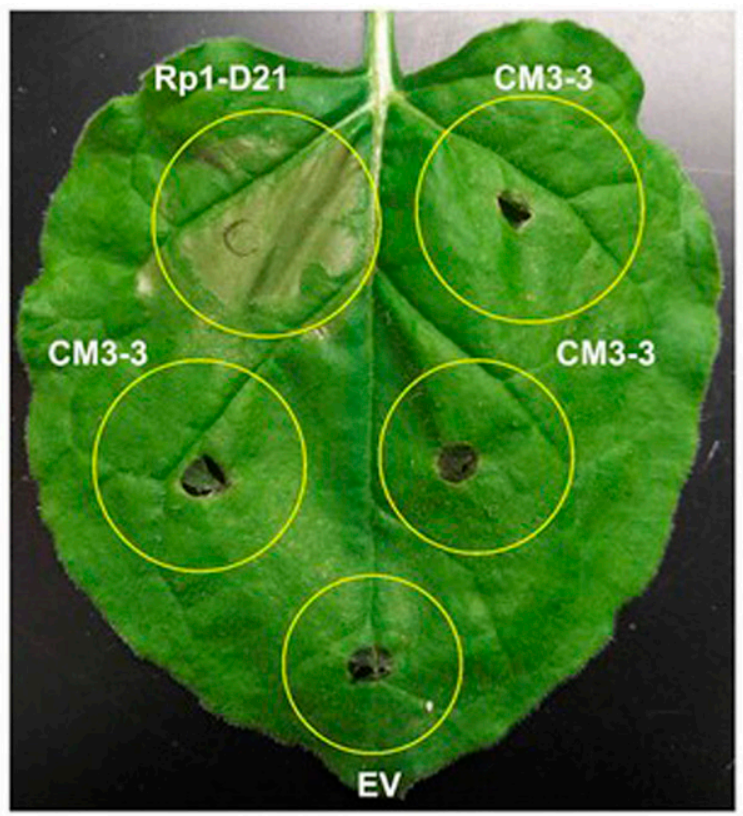

B

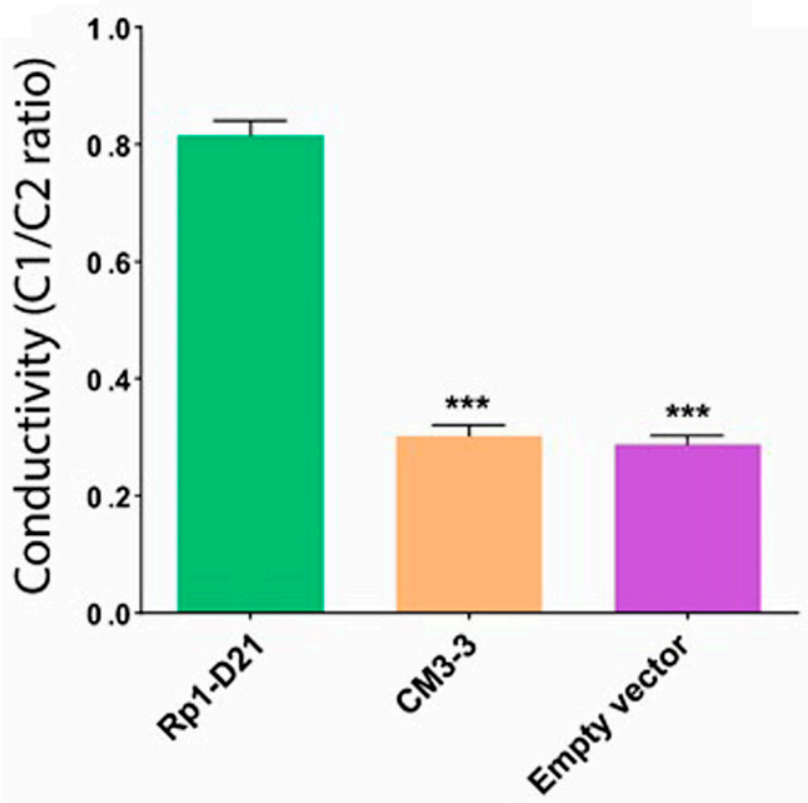

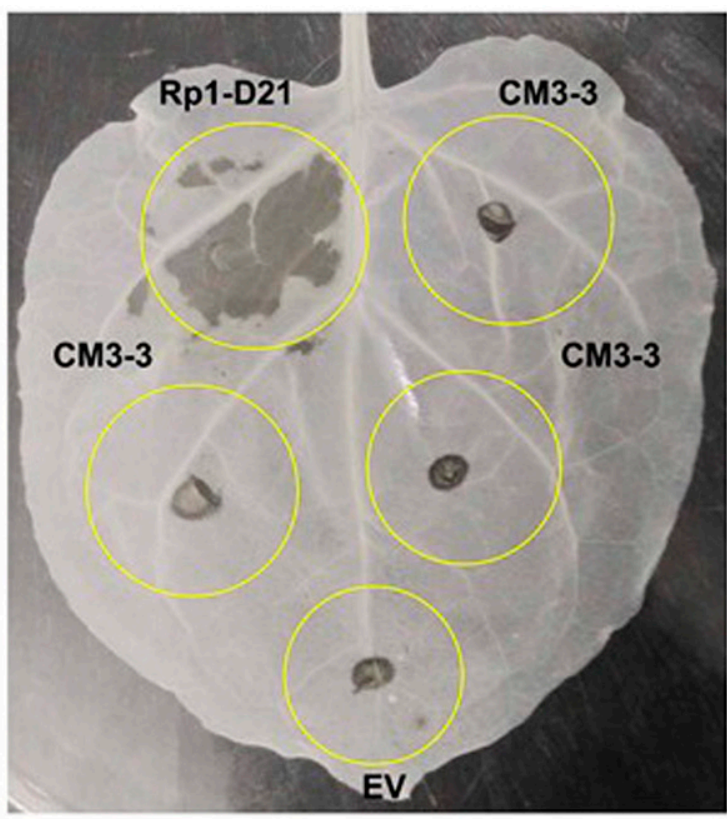

C

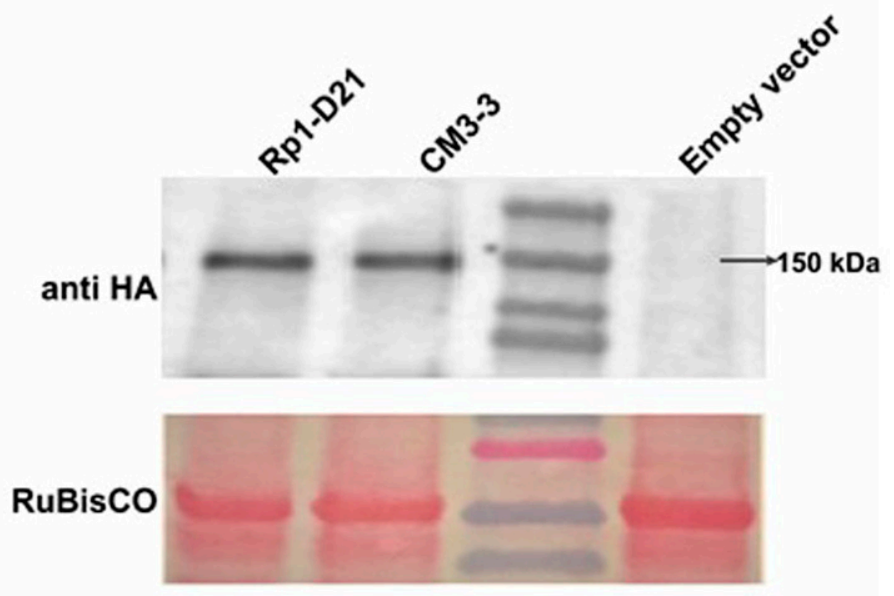

Fig. 4. Transient expression of Rp1-D21 from chimeric mutant (CM) CM3-3 in Nicotiana benthamiana. A, N. benthamiana leaf showing cell death caused by expression of the functional Rp1-D21 allele compared with the mutated CM3-3 Rp1-D21 allele whose expression does not induce cell death. $\mathrm{EV}=$ empty vector control $\mathbf{B}$, Ion leakage analysis shows lower conductivity ratio $(\mathrm{C} 1=$ initial conductivity/C2 $=$ final conductivity $)$, indicating less cell death from tissue in which CM3-3 Rp1-D21 allele was expressed compared with leaf tissue in which the functional Rp1-D21 clone was transiently expressed. C, Western blot analysis using anti-HA antibody shows that both Rp1-D21 proteins are expressed in the $N$. benthamiana heterologous system. Significance between treatments were analyzed using one-way analysis of variance: Three asterisks $(* * *)$ indicate $P<0.001$. This experiment was repeated three times with similar results. 
Protein analysis showed that each protein was successfully expressed (Fig. 4C).

HR-induced cell death is cell autonomous but some defense response transcript levels respond to a diffusible signal.

In order to look at other aspects of the Rp1-D21 phenotype, transcript levels of various markers of disease response were measured in the lesioned and nonlesioned sectors of CM1, $\mathrm{CM} 2$, and $\mathrm{CM} 3$ plants. In addition to the sectors, transcript abundance was also determined in isogenic Rp1-D21 and wildtype control plants grown under the same conditions and at the same time as the chimeric plants in neighboring rows or pots. $\mathrm{HR}$ is typically associated with a wider defense response that includes the upregulation of a suite of pathogeneses related (PR) genes (Ali et al. 2018). We were interested to determine whether the induction of PR gene expression was cell autonomous in a similar way to the HR, chlorosis and cell death-marker gene expression phenotypes.

As described above for the marker genes associated with cell death, we determined the transcript levels of three genes-pathogenesis-related 1 (ZmPRl), pathogenesis-related 4b (ZmPR4b), and pathogenesis-related protein 5 (ZmPR5) - in the lesioned and nonlesioned sectors of CM1, CM2, and CM3 and their corresponding isogenic nonchimeric lesioned and nonlesioned counterparts (Fig. 5). ZmPRI, ZmPR4b, and ZmPR5 have been previously shown to be induced during the defense response in maize (Chintamanani et al. 2010; Christensen et al. 2015) and ZmPRI and ZmPR5 were previously reported to be salicylic acid induced (Morris et al. 1998; Vargas et al. 2012).

ZmPR1, ZmPR4b, and ZmPR5 showed relatively higher expression levels in nonchimeric lesioned plants carrying the Rp1-D21 gene and in lesioned sectors of CM1, CM2, and CM3 chimeric plants than in their nonlesioned nonchimeric counterparts (Fig. 5). Of particular interest was the observation that expression levels of $Z m P R 4 b$ and $Z m P R 5$ were significantly higher in nonlesioned sectors of CM1 and CM2 chimeric plants compared with their nonlesioned nonchimeric counterparts, though this was not the case for CM3 (Fig. 5). The expression of ZmPR1 in the nonlesioned sectors of CM1 was significantly higher than in its nonlesioned nonchimeric counterpart but this was not the case for CM2 or CM3.

We also assessed the expression of $\mathrm{ZmCCoAOMT2} \mathrm{(Fig.} \mathrm{5),}$ which encodes a member of the family of enzymes that includes lignin biosynthetic enzyme caffeoyl-coenzyme A (CoA) O-methyltransferase. A previous study (Wang and Balint-Kurti 2016) showed that Rp1-D21-induced HR could be suppressed by ZmCCoAMT2 and that ZmCCoAMT2 transcript levels increased due to HR induced by $R p 1-D 21$. As expected, the $\mathrm{ZmCCOAOMT2}$ expression levels were significantly higher in all lesioned plants and sectors than in the nonlesioned controls (Fig. 5). In this case, expression in the nonlesioned sectors of $\mathrm{CM} 2$ (but not of CM1 and CM3) was significantly higher than in its nonlesioned nonchimeric counterpart.

Although not entirely consistent between plants and between genes, these results suggest that, whereas the HR and chlorosis phenotypes associated with $R p 1-D 21$ are cell autonomous, this is not true for some of the associated defense responses. It appears that a signal from the lesioned sector induces increased PR gene expression in the neighboring nonlesioned sectors. Although we have no direct evidence, it seems likely that this signal is salicylic acid or a close derivative. Betsuyaku et al. (2018) reported high expression of $P R 1$ and another defense marker gene, VSP1, in Arabidopsis leaves in the ring of cells surrounding cells undergoing an HR induced by the activation of the Rps2 NLR protein. They showed that these regions were relatively small (measuring less than $1 \mathrm{~mm}$ in width), that their borders were relatively well defined, and that each gene was
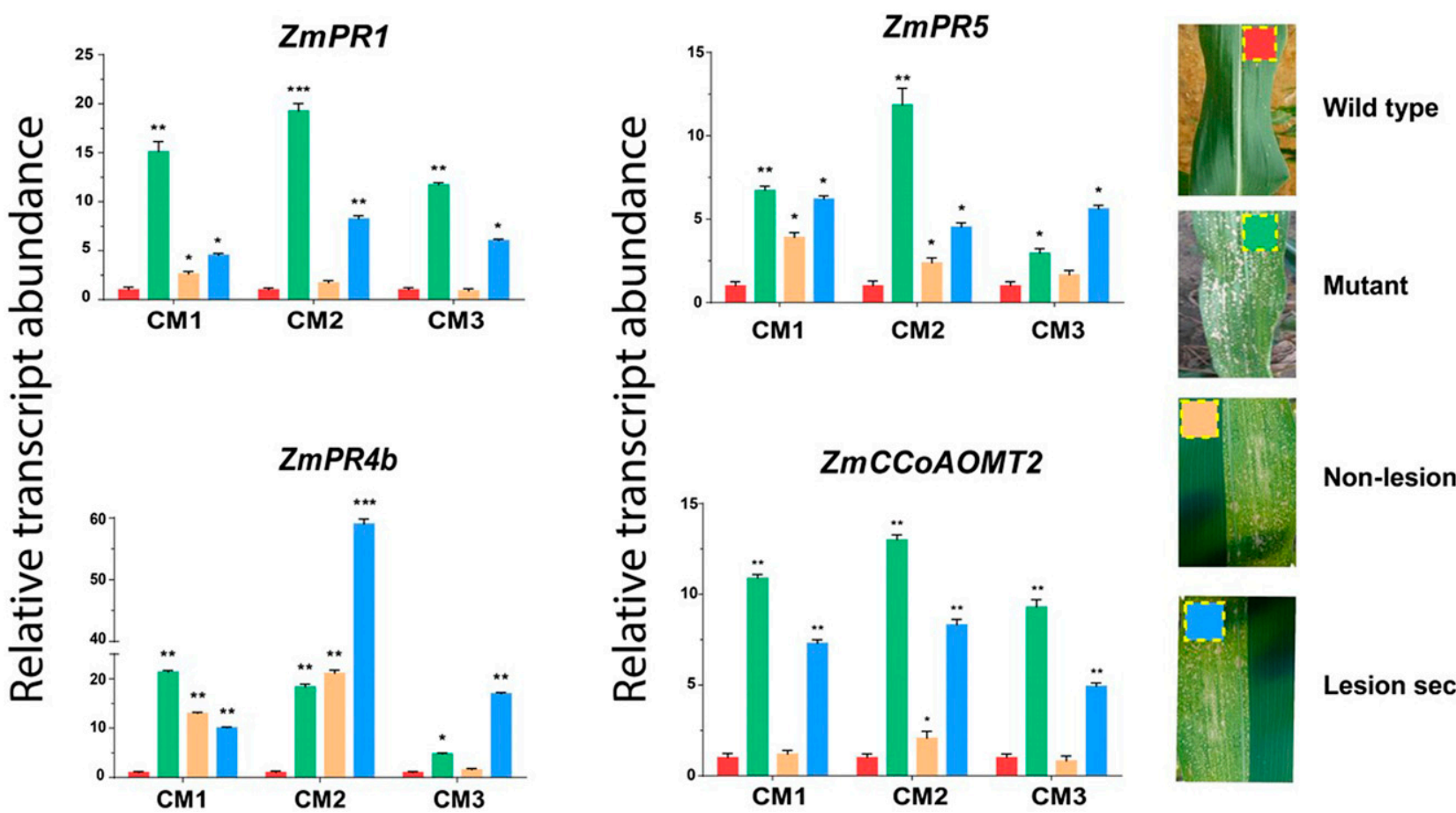

Fig. 5. Gene expression of defense-response marker genes in lesioned and nonlesioned sectors and plants. Quantitative reverse-transcription (qRT)-PCR of ZmPR1, ZmPR4b, ZmPR5, and ZmCCoAOMT2 genes in leaves of plants carrying Rp1-D21 (mutant); lesioned sectors of chimeric mutant 1 (CM1), $\mathrm{CM} 2$, and CM3 (lesioned sector) in wild-type plants lacking Rp1-D21 (wild type) and in nonlesioned sectors of CM1, CM2, and CM3 (nonlesioned sector). Histogram bars are color coded to indicate type of tissue. A visual key is shown on the right. Results displayed are the averages of all biological replicates in each case. For CM1 and CM2, three biological replicates we collected as individual leaves from the same plant as described. For CM3 qRT-PCR results, we considered samples from CM3-1 to CM3-3 to be three biological replicates. The error bar shows standard error. Significance between treatments were analyzed using Student's $t$ test; asterisks $\left({ }^{*},{ }^{*}\right.$, or ${ }^{* * *}$ ) indicate $P<0.05,0.01$, and 0.001 , respectively. 
expressed in a distinct spatiotemporal pattern. The data presented here are far less detailed with respect to patterns of gene expression but it should be noted that the levels of defense gene upregulation in the nonlesioned sectors might vary substantially, depending on how far they are from the lesioned sectors.

In summary, we suggest that cell death and chlorosis associated with the Rp1-D21 phenotype is cell autonomous, while another aspect (induction of PR genes) is non-cell autonomous. Freeling (1992) suggested that "It is entirely expected for dominant phenotypes to be cell autonomous for one component of phenotype and non-cell autonomous for another" and demonstrated that this was true for the maize $D 8$ dwarf allele. Our results suggest that $R p 1-D 21$ is necessary and sufficient for the observed spontaneous HR phenotype and are consistent with previous reports (Bennetzen et al. 1988). The fact that macroscopic spots of cell death occur on leaves and leaf sectors carrying RplD21 suggests that a "pro-death" signal must be produced by cells undergoing $\mathrm{HR}$ that predisposes neighboring cells also to undergo HR. However, Rpl-D21 is required to sensitize the neighboring cells so that they are competent to respond to this signal. In other systems, pathogen-induced HR can manifest as a single-cell phenotype in some cases or as macroscopic spots in others (Balint-Kurti 2019). Mutations affecting the autophagy system (Liu et al. 2005; Patel and Dinesh-Kumar 2008) or the production of reactive oxygen species (Dietrich et al. 1997) can lead to the unrestricted spread of cell death once initiated by activation of an NLR. Although HR is cell autonomous in this case, we should be cautious in assuming that this is universally true for HR initiated by all NLRs. The interaction between processes facilitating the spread of HR cell death, which may enhance resistance to biotrophic pathogens, and processes that contain it, which may reduce the negative growth aspects of HR, has not been clearly elucidated and is likely to be quite complex, reflecting aspects of the plant-pathogen evolutionary arms race.

\section{MATERIALS AND METHODS}

\section{Genetic material and mutagenesis.}

Eleven mutants, with leaves chimeric for $R p 1-D 21$ derived from four independent populations, were used for this study. CM1 was a spontaneous mutant identified in the field and was derived from a cross between the maize lines Tx303 and H95:Rp1-D21 (the maize line H95 carrying Rpl-D2lin a heterozygous state). CM2 was a spontaneous mutant identified in the field and was derived from a cross between the maize lines M37 and H95:Rp1-D21. Both CM1 and CM2 were identified in the same field in Clayton, NC in summer 2017.

CM3-1 CM3-2, and CM3-3 were derived from a set of B73:Rp1-D21\#4 plants (the maize line B73 carrying an allele of Rp1-D21 called Rp1-D21\#4 in a heterozygous state), which were grown from seed mutagenized using EMS. The protocol for EMS seed mutagenesis was as follows: 500 seeds were soaked in $500 \mathrm{ml}$ of $20 \mathrm{mM}$ EMS for $6 \mathrm{~h}$ in darkness, then rinsed in running water for $8 \mathrm{~h}$. The seed were dried and planted in a greenhouse at Purdue University (West Lafayette, IN, U.S.A.). The plants were grown at $25^{\circ} \mathrm{C}$ with a cycle of $16 \mathrm{~h} /$ day and $8 \mathrm{~h}$ /night. Among 500 plants grown in the greenhouse facility, three plants (CM3-1, CM3-2, and CM3-3) were identified with chimeric wild-type sectors in their leaves.

CM4-1, CM4-5, CM4-6, CM4-10, CM4-12, and CM4-13 were derived from a set of H95:Rp1-D21 plants (the maize line H95 carrying Rp1-D21 in a heterozygous state), which were grown from seed mutagenized using EMS using the same protocol as described above. The seed were dried and planted in field at Clayton, NC in summer 2019. Among 1,100 plants, 18 plants showed chimeric wild-type sectors, among which six plants (CM4-1,
CM4-5, CM4-6, CM4-10, CM4-12, and CM4-13) displaying the clearest sectors were selected for further studies.

\section{Sample collection.}

Multiple leaves displayed Rp1-D21 sectors on the CM1 and CM2 plants. For each plant, $5-\mathrm{cm}^{2}$ samples of tissue were excised from lesioned sectors and nonlesioned sectors. The closest edge of sampled tissue square was approximately $0.5 \mathrm{~cm}$ from the sector boundary in each case. Samples were collected from three individual leaves of the same plant and are considered as biological replicates. Because all of the leaves in the plant showed chimeric sectors, samples were collected from leaf four, the ear leaf, and the flag leaf. Similarly, leaf samples from leaf four, the ear leaf, and the flag leaf were collected from three different (nonchimeric) mutant and wild-type plants from Tx303 $\times$ H95:Rp1-D21 and M37W $\times$ H95:Rp1-D21 families and used as isogenic controls for $\mathrm{CM} 1$ and $\mathrm{CM} 2$, respectively. All of the samples were collected from 70-day-old plants.

Samples were collected from the lesioned and nonlesioned sectors of 4-week-old CM3-1, CM3-2, and CM3-3 plants, the three EMS-treated B73:Rp1-D21\#4 plants displaying chimeric sectors. The near edge of the sampled tissue was approximately $2 \mathrm{~mm}$ away from the sector border in each case. The distance between borders is smaller in CM3 compared with CM1 and $\mathrm{CM} 2$, because the CM3 plants were younger and their leaves were considerably smaller. Additionally, samples were collected from three wild-type (B73) and three lesioned (B73:Rp1-D21\#4) plants as controls. All samples were collected 4 weeks after planting, frozen in liquid nitrogen immediately, and stored at $-80^{\circ} \mathrm{C}$ until further use.

Samples were collected from the lesioned and nonlesioned sectors of 7-week-old CM4-1, CM4-5, CM4-6, CM4-10, CM412, and CM4-13 plants, six EMS-treated H95:Rp1-D21 plants displaying chimeric sectors. For each plant, $5-\mathrm{cm}^{2}$ samples of tissue were excised from lesioned sectors and nonlesioned sectors. The closest edge of sampled tissue square was approximately $0.5 \mathrm{~cm}$ from the sector boundary in each case. All of the samples were collected, frozen in liquid nitrogen immediately, and stored at $-80^{\circ} \mathrm{C}$ until further use.

\section{Total RNA isolation and amplification of $R p 1-D 21$ coding sequence.}

Ten 6-mm-diameter leaf punches from the samples described above were used to isolate RNA. Total RNA was extracted from maize leaf samples with an RNeasy Plant Mini Kit (Qiagen) and treated with RNase-free DNase I (Thermo Fisher Scientific) to remove residual genomic DNA, according to the manufacturer's protocol. Complementary DNA (cDNA) was synthesized from $3 \mu \mathrm{g}$ of total RNA with RevertAid reverse transcription (Thermo Fisher Scientific) with OligodT primers, according to the manufacturer's protocol.

Primers Rp1D21F1 and Rp1D21R1 (Supplementary Table $\mathrm{S} 1)$ were used to amplify the Rp1-D21 coding sequence with Q5 high-fidelity DNA polymerase (M0491; New England Biolabs). The reaction mixture and conditions for PCR were followed according to the manufacturer's protocol. A second primer set (Rp1D21F2 and Rp1D21R2) (Supplementary Table S1) was used to amplify the Rpl-D21 coding sequence with Q5 high-fidelity DNA polymerase (M0491; New England Biolabs). The product derived from the Rp1D21F2/R2 amplification was used exclusively for Sanger sequencing.

Both sets of primers that amplify Rpl-D21 were validated using H95:Rp1-D21 and B73:Rp1-D21 as positive control templates and DNA from B73, Tx303, H95, and M37W as negative control templates. 


\section{Expression analysis using qRT-PCR.}

Primers for qRT-PCR were first used to generate standard PCR products, which were then sequenced using standard Sanger sequencing to confirm that the expected gene fragments were amplified. qRT-PCR was carried out on a CFX384 realtime PCR detection system (Bio-Rad) with SsoAdvanced SYBR Green Master mix (Bio-Rad), according to the manufacturer's instructions. The primers used for qRT-PCR studies are provided in Supplementary Table S1. Gene expression was measured relative to the housekeeping gene $Z m G A P D H 1$. The $2^{-\triangle \Delta C T}$ method (normalized to ZmGAPDH1 expression and relative to a wild-type control) was used to calculate relative gene expression (Livak and Schmittgen 2001). Three technical replicates were performed for each sample. Three individual leaves in CM1 and CM2 were considered as three biological replicates. In the case of CM3, samples from CM3-1, CM3-2, and CM3-3 were considered as biological replicates. Statistical analysis was calculated using Student's $t$ test (GraphPad PRISM).

\section{Sequencing and assembly of Rp1-D21 cDNA.}

Two different approaches were used for sequencing. The RplD21 PCR products derived from amplification using the Rp1D21F1/R1 primers on DNA derived from CM1, CM2, CM3-1, CM3-2, and CM3-3 plants were purified using a DNA Clean \& Concentrator-5 kit (Zymo Research). The purified PCR products were quantified using a Nanodrop 2000/2000c spectrophotometer (Thermo Fisher Scientific). The PCR amplicon was sequenced by a Wideseq methodology, as detailed by Khangura et al. (2019). Briefly, sequencing libraries were generated using a hyperactive variant of Tn5 transposase using the Illumina Nextera DNA library preparation kit. The multiplexed libraries were sequenced using the MiSeq instrument (Illumina) to generate the paired-end reads $(2 \times 250 \mathrm{bp})$ at the Purdue Genomics Core Facility. Paired-end reads from each sample were processed to remove adapters and poor-quality bases $(<$ Phred-20) using Trimmomatic (Bolger et al. 2014) with the following settings: LEADING:20 TRAILING:20 MINLEN:30. Only the filtered paired reads were used for further processing. The filtered paired-end reads were aligned to the Rpl-D21 reference mRNA sequence (NCBI accession number KF951062.1) using short-read aligner BWA version 0.7.12 (Li and Durbin 2009). The alignments were generated using the BWA sample command with default parameters to generate Sequence Alignment/Map (SAM) files. The SAM files were converted into Binary Alignment Map (BAM) files, followed by sorting, indexing, and finally variant calling using the mpileup function in SAMtools suite version 1.3 ( $\mathrm{Li}$ et al. 2009). The detected variants were manually inspected by visualizing each sample in IGV version 2.8.2 (Robinson et al. 2011) using the sorted BAM files. Mean coverage across the reference-aligned reads for each sample were calculated using the depth function in SAMtools (Table 1; Supplementary Table S2). All derived sequences have been deposited in GenBank (Supplementary Table S3). Multiple sequence alignments were performed using MEGA software (Kumar et al. 2004).

The second sequencing approach, Sanger sequencing, was performed on the Rp1-D21 PCR products derived from amplification using the Rp1D21F2/R2 primers. PCR products were purified using a DNA Clean \& Concentrator-5 kit (Zymo Research) and quantified using a Nanodrop 2000/2000c spectrophotometer (Thermo Fisher Scientific). They were cloned into pGEM-T vector (Promega Corp.) following the manufacturer's protocol. Two independent colonies for each recombinant clone were Sanger sequenced. The full-length sequence $(3,873 \mathrm{bp})$ was achieved using seven nested primers (Supplementary Table $\mathrm{S} 1$, primers 15 to 25$)$. The multiple sequence alignments shown in Supplementary Figures S1 to S4 were performed using Geneious Prime software.

\section{Cloning and transient expression in $N$. benthamiana.}

The purified PCR products of Rp1-D21 amplified from CM3-3 and from "normal" Rp1-D21 plants were cloned into pCR8/GW/ TOPO TA vector following the manufacturer's protocol (Thermo Fisher Scientific). The resultant ligation mixtures were transformed into Escherichia coli, and colony PCR was performed to identify positive clones. The positive recombinant clone for each gene was further subcloned into the pGWB614 vector carrying the $3 \mathrm{xHA}$ tag at the $\mathrm{C}$ terminal using the gateway cloning protocol (Thermo Fisher Scientific). The resulting recombinant clone was further transformed into Agrobacterium tumefaciens for downstream applications.

Wild-type $N$. benthamiana plants were grown in the laboratory at $25^{\circ} \mathrm{C}$ with a cycle of $16 \mathrm{~h}$ of light and $8 \mathrm{~h}$ of darkness. A. tumefaciens GV3101 (pMP90) carrying the Rp1-D21 expression constructs in the binary vector was grown at $28^{\circ} \mathrm{C}$ overnight in $50 \mathrm{ml}$ of Luria-Bertani media with appropriate antibiotics. The culture was centrifuged at 4,000 rpm for $30 \mathrm{~min}$ at $4{ }^{\circ} \mathrm{C}$. The pellet was resuspended in infiltration buffer diluted to a final concentration of optical density at $600 \mathrm{~nm}\left(\mathrm{OD}_{600}\right)=$ 0.6 and was combined with a p19 (Lakatos et al. 2004) silencing suppressor construct at $\mathrm{OD}_{600}=0.3$. The resultant mixture was used for transient expression in $N$. benthamiana. The detailed procedures were performed according to Wang et al. (2015b).

\section{Protein analysis and Ion leakage.}

For protein expression analysis, at least three leaf discs $(1.2$ $\mathrm{mm}$ in diameter) from each leaf were collected at $30 \mathrm{hpi}$. Total protein was isolated using $300 \mu \mathrm{l}$ of protein extraction buffer $(20$ mM Tris- $\mathrm{HCl}$ [pH 8.0], $150 \mathrm{mM} \mathrm{NaCl}, 1 \mathrm{mM}$ EDTA [pH 8.0], $1 \%$ Triton X-100, $0.1 \%$ sodium dodecyl sulfate [SDS], $10 \mathrm{mM}$

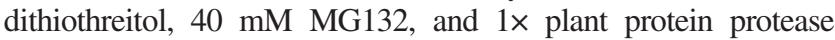
inhibitor mixture) (Sigma-Aldrich). Samples were centrifuged at $14,000 \mathrm{rpm}$ for $10 \mathrm{~min}$ at $4^{\circ} \mathrm{C}$, and $50 \mu \mathrm{l}$ of supernatant was mixed with $2 \times$ Laemmeli sample buffer at a 1:1 ratio. The mixture was boiled at $95^{\circ} \mathrm{C}$ for $5 \mathrm{~min}$ and samples were used for SDS-polyacrylamide gel electrophoresis. The protein gel was transferred to nitrocellulose membrane and was incubated with anti-HA antibody conjugated with horseradish peroxidase (HRP) at 1:350 dilution (Roche). The HRP signal was detected using SuperSignal West Femto Maximum Sensitivity Substrate (Thermo Scientific).

In total, five leaf discs (2 $\mathrm{mm}$ in diameter) from different plants were used for measuring ion leakage. The detailed procedures were performed according to Wang et al. (2015b). The ion leakage was calculated using the $\mathrm{C} 1 / \mathrm{C} 2$ ratio (where $\mathrm{C} 1=$ initial conductivity and $\mathrm{C} 2$ = final conductivity). The statistical analysis was calculated using one-way analysis of variance (GraphPad PRISM).

\section{ACKNOWLEDGMENTS}

We thank C. Herring and the staff at Central Crops Research Station for care of our field trials, G. Marshall for technical assistance, and H. Wilson and the North Carolina State University Greenhouse staff.

\section{LITERATURE CITED}

Ali, S., Ganai, B. A., Kamili, A. N., Bhat, A. A., Mir, Z. A., Bhat, J. A., Tyagi, A., Islam, S. T., Mushtaq, M., Yadav, P., Rawat, S., and Grover, A. 2018. Pathogenesis-related proteins and peptides as promising tools for engineering plants with multiple stress tolerance. Microbiol. Res. 212-213:29-37.

Balint-Kurti, P. 2019. The plant hypersensitive response: Concepts, control and consequences. Mol. Plant Pathol. 20:1163-1178. 
Becraft, P. W., Bongard-Pierce, D. K., Sylvester, A. W., Poethig, R. S., and Freeling, M. 1990. The liguleless- 1 gene acts tissue specifically in maize leaf development. Dev. Biol. 141:220-232.

Bennetzen, J. L., Blevins, W. E., and Ellingboe, A. H. 1988. Cell-autonomous recognition of the rust pathogen determines $R p 1$-specified resistance in maize. Science 241:208-210.

Betsuyaku, S., Katou, S., Takebayashi, Y., Sakakibara, H., Nomura, N., and Fukuda, H. 2018. Salicylic acid and jasmonic acid pathways are activated in spatially different domains around the infection site during effector-triggered immunity in Arabidopsis thaliana. Plant Cell Physiol. 59:8-16.

Bolger, A. M., Lohse, M., and Usadel, B. 2014. Trimmomatic: A flexible trimmer for Illumina sequence data. Bioinformatics 30:2114-2120.

Browder, L. E. 1972. Designation of two genes for resistance to Puccinia recondita in Triticum aestivum 1 . Crop Sci. 12:705-706.

Chaikam, V., Negeri, A., Dhawan, R., Puchaka, B., Ji, J., Chintamanani, S., Gachomo, E. W., Zillmer, A., Doran, T., Weil, C., Balint-Kurti, P., and Johal, G. 2011. Use of mutant-assisted gene identification and characterization (MAGIC) to identify novel genetic loci that modify the maize hypersensitive response. Theor. Appl. Genet. 123:985-997.

Chang, C., Yu, D., Jiao, J., Jing, S., Schulze-Lefert, P., and Shen, Q.-H. 2013. Barley MLA immune receptors directly interfere with antagonistically acting transcription factors to initiate disease resistance signaling. Plant Cell 25:1158-1173.

Chintamanani, S., Hulbert, S. H., Johal, G. S., and Balint-Kurti, P. J. 2010. Identification of a maize locus that modulates the hypersensitive defense response, using mutant-assisted gene identification and characterization. Genetics 184:813-825.

Christensen, S. A., Huffaker, A., Kaplan, F., Sims, J., Ziemann, S., Doehlemann, G., Ji, L., Schmitz, R. J., Kolomiets, M. V., Alborn, H. T., Mori, N., Jander, G., Ni, X., Sartor, R. C., Byers, S., Abdo, Z., and Schmelz, E. A. 2015. Maize death acids, 9-lipoxygenase-derived cyclopente(a)nones, display activity as cytotoxic phytoalexins and transcriptional mediators. Proc. Natl. Acad. Sci. U.S.A. 112:11407-11412.

Dangl, J. L., and Jones, J. D. G. 2019. A pentangular plant inflammasome. Science 364:31-32.

Dietrich, R. A., Richberg, M. H., Schmidt, R., Dean, C., and Dangl, J. L. 1997. A novel zinc finger protein is encoded by the Arabidopsis LSDI gene and functions as a negative regulator of plant cell death. Cell 88:685-694.

Dropkin, V. H. 1969. The necrotic reaction of tomatoes and other hosts resistant to Meloidogyne: Reversal by temperature. Phytopathology 59:1632-1637.

Flor, H. H. 1942. Inheritance of pathogenicity in Melampsora lini. Phytopathology 32:653-669.

Freeling, M. 1992. A conceptual framework for maize leaf development. Dev. Biol. 153:44-58.

Fyvie, M. J., Murray, J. A. H., and Kilby, N. J. 2000. Mosaic analysis of GL2 gene expression and cell layer autonomy during the specification of Arabidopsis leaf trichomes. Genesis 28:68-74.

He, Y., Karre, S., Johal, G. S., Christensen, S. A., and Balint-Kurti, P. 2019a. A maize polygalacturonase functions as a suppressor of programmed cell death in plants. BMC Plant Biol. 19:310.

He, Y., Kim, S.-B., and Balint-Kurti, P. 2019b. A maize cytochrome b-c1 complex subunit protein ZmQCR7 controls variation in the hypersensitive response. Planta 249:1477-1485.

Hogenhout, S. A., Van der Hoorn, R. A. L., Terauchi, R., and Kamoun, S. 2009. Emerging concepts in effector biology of plant-associated organisms. Mol. Plant-Microbe Interact. 22:115-122.

Horsefield, S., Burdett, H., Zhang, X., Manik, M. K., Shi, Y., Chen, J., Qi, T., Gilley, J., Lai, J.-S., Rank, M. X., Casey, L. W., Gu, W., Ericsson, D. J., Foley, G., Hughes, R. O., Bosanac, T., von Itzstein, M., Rathjen, J. P., Nanson, J. D., Boden, M., Dry, I. B., Williams, S. J., Staskawicz, B. J., Coleman, M. P., Ve, T., Dodds, P. N., and Kobe, B. 2019. $\mathrm{NAD}^{+}$cleavage activity by animal and plant TIR domains in cell death pathways. Science 365:793-799.

Hu, G., Richter, T. E., Hulbert, S. H., and Pryor, T. 1996. Disease lesion mimicry caused by mutations in the rust resistance gene $r p 1$. Plant Cell 8:1367-1376.

Hulbert, S. H. 1997. Structure and evolution of the rpl complex conferring rust resistance in maize. Annu. Rev. Phytopathol. 35:293-310.

Hulbert, S. H., and Bennetzen, J. L. 1991. Recombination at the Rp1 locus of maize. Mol. Gen. Genet. 226:377-382.

Jones, D. R., and Deverall, B. J. 1978. The use of leaf transplants to study the cause of hypersensitivity to leaf rust, Puccinia recondita, in wheat carrying the Lr20 gene. Physiol. Plant Pathol. 12:311-314.
Khangura, R. S., Marla, S., Venkata, B. P., Heller, N. J., Johal, G. S., and Dilkes, B. P. 2019. A Very oil yellow1 modifier of the Oil yellow1-N1989 allele uncovers a cryptic phenotypic impact of cis-regulatory variation in maize. G3-Genes Genom. Genet. 9:375-390.

Kim, Y., Schumaker, K. S., and Zhu, J. K. 2006. EMS mutagenesis of Arabidopsis. Methods Mol. Biol. 323:101-103.

Klapacz, J., Pottenger, L. H., Engelward, B. P., Heinen, C. D., Johnson, G. E., Clewell, R. A., Carmichael, P. L., Adeleye, Y., and Andersen, M. E. 2016. Contributions of DNA repair and damage response pathways to the non-linear genotoxic responses of alkylating agents. Mutat. Res. Rev. Mutat. Res. 767:77-91.

Kourelis, J., and van der Hoorn, R. A. L. 2018. Defended to the nines: 25 Years of resistance gene cloning identifies nine mechanisms for $\mathrm{R}$ protein function. Plant Cell 30:285-299.

Kumar, S., Tamura, K., and Nei, M. 2004. MEGA3: Integrated software for molecular evolutionary genetics analysis and sequence alignment. Brief. Bioinf. 5:150-163.

Lakatos, L., Szittya, G., Silhavy, D., and Burgyán, J. 2004. Molecular mechanism of RNA silencing suppression mediated by p19 protein of tombusviruses. EMBO J. 23:876-884.

Li, H., and Durbin, R. 2009. Fast and accurate short read alignment with Burrows-Wheeler transform. Bioinformatics 25:1754-1760.

Li, H., Handsaker, B., Wysoker, A., Fennell, T., Ruan, J., Homer, N., Marth, G., Abecasis, G., and Durbin, R.; 1000 Genome Project Data Processing Subgroup. 2009. The Sequence Alignment/Map format and SAMtools. Bioinformatics 25:2078-2079.

Liu, Y., Schiff, M., Czymmek, K., Tallóczy, Z., Levine, B., and DineshKumar, S. P. 2005. Autophagy regulates programmed cell death during the plant innate immune response. Cell 121:567-577.

Livak, K. J., and Schmittgen, T. D. 2001. Analysis of relative gene expression data using real-time quantitative PCR and the $2^{-\Delta \Delta C T}$ method. Methods 25:402-408.

Ma, S., Lapin, D., Liu, L., Sun, Y., Song, W., Zhang, X., Logemann, E., Yu, D., Wang, J., Jirschitzka, J., Han, Z., Schulze-Lefert, P., Parker, J. E. and Chai, J. 2020. Direct pathogen-induced assembly of an NLR immune receptor complex to form a holoenzyme. Science 370:eabe3069.

Ma, W., Westmoreland, J. W., Gordenin, D. A., and Resnick, M. A 2011. Alkylation base damage is converted into repairable doublestrand breaks and complex intermediates in G2 cells lacking AP endonuclease. PLoS Genet. 7:e1002059.

Martin, R., Qi, T., Zhang, H., Liu, F., King, M., Toth, C., Nogales, E., and Staskawicz, B. J. 2020. Structure of the activated Roq1 resistosome directly recognizing the pathogen effector XopQ. Science 370:eabd9993.

Morris, S. W., Vernooij, B., Titatarn, S., Starrett, M., Thomas, S., Wiltse, C. C., Frederiksen, R. A., Bhandhufalck, A., Hulbert, S., and Uknes, S. 1998. Induced resistance responses in maize. Mol. Plant-Microbe Interact. 11:643-658

Mur, L. A., Kenton, P., Lloyd, A. J., Ougham, H., and Prats, E. 2008. The hypersensitive response; the centenary is upon us but how much do we know? J. Exp. Bot. 59:501-520.

Murphree, C., Kim, S.-B., Karre, S., Samira, R., and Balint-Kurti, P. 2020. Use of virus-induced gene silencing to characterize genes involved in modulating hypersensitive cell death in maize. Mol. Plant Pathol. 21:1662-1676.

Negeri, A., Wang, G. F., Benavente, L., Kibiti, C. M., Chaikam, V., Johal, G., and Balint-Kurti, P. 2013. Characterization of temperature and light effects on the defense response phenotypes associated with the maize Rp1-D21 autoactive resistance gene. BMC Plant Biol. 13:106.

Olukolu, B. A., Negeri, A., Dhawan, R., Venkata, B. P., Sharma, P., Garg, A., Gachomo, E., Marla, S., Chu, K., Hasan, A., Ji, J., Chintamanani, S., Green, J., Shyu, C.-R., Wisser, R., Holland, J., Johal, G., and Balint-Kurti, P. 2013. A connected set of genes associated with programmed cell death implicated in controlling the hypersensitive response in maize. Genetics 193:609-620.

Olukolu, B. A. B. A., Wang, G.-F. G.-F., Vontimitta, V., Venkata, B. P. B. P. B. P., Marla, S., Ji, J., Gachomo, E., Chu, K., Negeri, A., Benson, J., Nelson, R., Bradbury, P., Nielsen, D., Holland, J. B. J. B., Balint-Kurti, P. J. P. J. P. J., and Johal, G. 2014. A genome-wide association study of the maize hypersensitive defense response identifies genes that cluster in related pathways. PLoS Genet. 10:e1004562.

Patel, S., and Dinesh-Kumar, S. P. 2008. Arabidopsis ATG6 is required to limit the pathogen-associated cell death response. Autophagy 4:20-27.

Pontier, D., Gan, S., Amasino, R. M., Roby, D., and Lam, E. 1999. Markers for hypersensitive response and senescence show distinct patterns of expression. Plant Mol. Biol. 39:1243-1255. 
Robinson, J. T., Thorvaldsdóttir, H., Winckler, W., Guttman, M., Lander, E. S., Getz, G., and Mesirov, J. P. 2011. Integrative genomics viewer. Nat. Biotechnol. 29:24-26.

Rossi, M., Goggin, F. L., Milligan, S. B., Kaloshian, I., Ullman, D. E., and Williamson, V. M. 1998. The nematode resistance gene $M i$ of tomato confers resistance against the potato aphid. Proc. Natl. Acad. Sci. U.S.A. 95:9750-9754.

Sato, M., and Gaul, H. 1967. Effect of ethyl methanesulfonate on the fertility of barley. Radiat. Bot. 7:7-10.

Scanlon, M. J., and Freeling, M. 1997. Clonal sectors reveal that a specific meristematic domain is not utilized in the maize mutant narrow sheath. Dev. Biol. 182:52-66.

Sega, G. A. 1984. A review of the genetic effects of ethyl methanesulfonate. Mutat. Res. 134:113-142.

Sudupak, M. A., Bennetzen, J. L., and Hulbert, S. H. 1993. Unequal exchange and meiotic instability of disease-resistance genes in the Rp1 region of maize. Genetics 133:119-125.

Takezawa, D. 2000. A rapid induction by elicitors of the mRNA encoding CCD-1, a $14 \mathrm{kDa} \mathrm{Ca} 2+$-binding protein in wheat cultured cells. Plant Mol. Biol. 42:807-817.

Tian, L., and Li, X. 2020. Enzyme formation by immune receptors. Science 370:1163-1164.

Vargas, W. A., Martín, J. M., Rech, G. E., Rivera, L. P., Benito, E. P., Díaz-Mínguez, J. M., Thon, M. R., and Sukno, S. A. 2012. Plant defense mechanisms are activated during biotrophic and necrotrophic development of Colletotrichum graminicola in maize. Plant Physiol. 158:1342-1358.

Virdi, S. K., Liu, Z., Overlander, M. E., Zhang, Z., Xu, S. S., Friesen, T. L., and Faris, J. D. 2016. New insights into the roles of host genenecrotrophic effector interactions in governing susceptibility of durum wheat to tan spot and Septoria nodorum blotch. G3-Genes Genom. Genet. 6:4139-4150.

Wagner, E. D., Anderson, D., Dhawan, A., Rayburn, A. L., and Plewa, M. J. 2003. Evaluation of EMS-induced DNA damage in the single cell gel electrophoresis (Comet) assay and with flow cytometric analysis of micronuclei. Teratog. Carcinog. Mutagen. 23:1-11.

Wan, L., Essuman, K., Anderson, R. G., Sasaki, Y., Monteiro, F., Chung, E.-H., Osborne Nishimura, E., DiAntonio, A., Milbrandt, J., Dangl, J. L., and Nishimura, M. T. 2019. TIR domains of plant immune receptors are NAD+-cleaving enzymes that promote cell death. Science 365:799-803.

Wang, G.-F., and Balint-Kurti, P. J. 2015. Cytoplasmic and nuclear localizations are important for the hypersensitive response conferred by maize autoactive Rp1-D21 protein. Mol. Plant-Microbe Interact. 28:1023-1031.

Wang, G.-F., and Balint-Kurti, P. J. 2016. Maize homologs of CCoAOMT and HCT, two key enzymes in lignin biosynthesis, form complexes with the NLR Rp1 protein to modulate the defense response. Plant Physiol. 171:2166-2177.

Wang, G.-F., He, Y., Strauch, R., Olukolu, B., Nielsen, D., Li, X., and Balint-Kurti, P. 2015a. Maize homologs of hydroxycinnamoyltransferase, a key enzyme in lignin biosynthesis, bind the nucleotide binding leucine-rich repeat Rp1 proteins to modulate the defense response. Plant Physiol. 169:2230-2243.

Wang, G.-F., Ji, J., El-Kasmi, F., Dangl, J. L., Johal, G., and Balint-Kurti, P. J. 2015b. Molecular and functional analyses of a maize autoactive NBLRR protein identify precise structural requirements for activity. PLoS Pathog. 11:e1004674.

Wang, J. J., Hu, M., Wang, J. J., Qi, J., Han, Z., Wang, G., Qi, Y., Wang, H.-W. W., Zhou, J.-M. M., and Chai, J. 2019a. Reconstitution and structure of a plant NLR resistosome conferring immunity. Science 364:eaav5870.

Wang, J. J., Wang, J. J., Hu, M., Wu, S., Qi, J., Wang, G. G., Han, Z., Qi, Y., Gao, N., Wang, H.-W. W., Zhou, J.-M. M., and Chai, J. 2019 b. Ligand-triggered allosteric ADP release primes a plant NLR complex. Science 364:eaav5868.

Yeom, S. I., Seo, E., Oh, S. K., Kim, K. W., and Choi, D. 2012. A common plant cell-wall protein HyPRP1 has dual roles as a positive regulator of cell death and a negative regulator of basal defense against pathogens. Plant J. 69:755-768. 